\title{
PROTON DRIVER BEAM INSTRUMENTATION
}

\author{
J.Crisp, B.Webber, FNAL, USA \\ V.Terekhov, IHEP, Protvino, Russia
}

\section{Abstract}

The multipurpose Fermilab Proton Driver is designed to provide short proton bunches at intensity $3 \times 10^{13}$ protons and at $15 \mathrm{~Hz}$ repetition rate. This presentation describes Beam Instrumentation foreseen both for the PD ring and its adjacent beam lines. The basic requirements and specifications of all beam instruments are given.

\section{INTRODUCTION}

The Fermilab Proton Driver complex will include a new $16 \mathrm{GeV}$ rapid cycling synchrotron, a new $400 \mathrm{MeV}$ Injection Line, and a new $16 \mathrm{GeV}$ Extraction Line with a majority of existing $8 \mathrm{GeV}$ Booster enclosure. The Proton Driver is designed to provide short bunches of $16 \mathrm{GeV}$ at total intensity $3 \times 10^{13} \mathrm{p}$ and repetition rate $15 \mathrm{~Hz}$. That will result in a high beam power of $1.2 \mathrm{MW}$. Such a package of machine parameters requires significant beam instrumentation. High vacuum, beam intensity, repetitioe rate and radiation environment, very short bunches, ths range of changing RF and beam sizes present challengey for the beam instrumentation. At the same time man devices used at the FNAL Booster will be accepted.

\section{BEAM PROPERTIES AND SPECIAL REQUIREMENTS}

The Instrumentation will cope with beams:

- In the $400 \mathrm{MeV}$ Injection Line (320 m long): $\mathrm{H}^{-}$beam is modulated at $201.25 \mathrm{MHz}$ and contains $3.4 \times 10^{13}$ ions in a $90 \mu$ s pulse repeated at $15 \mathrm{~Hz}$. The beam will be chopped at 7.5 MHz in Stage 2 operation.

- In the Synchrotron:

Proton beam momentum changes from $400 \mathrm{MeV}$ to $16 \mathrm{GeV}$ with rotation periods changing from 3.3 to $2.4 \mu \mathrm{s}$. The $3 \times 10^{13}$ protons will be divided into 126$53 \mathrm{MHz} 12 \mathrm{GeV}$ bunches in Stage 1 and 18 - 7.5 $\mathrm{MHz} 16 \mathrm{GeV}$ bunches in Stage 2 operation.

Normalized transverse emittance is $60 \pi \mathrm{mm}$-mrad. Beam losses must not exceed $10 \%$ at injection or $1 \%$ extraction.

- In the $16 \mathrm{GeV}$ Extraction Line:

Single turn $(2.4 \mu \mathrm{s})$ fast extracted beam of $3 \times 10^{13}$ protons divided into 18 (or 126) bunches with an rms bunch length $(\sigma)$ of $1 \mathrm{~ns}$.

- A pilot beam with 20 times lower intensity will be used to verify injection, acceleration and extraction efficiency. That requires enlarged dynamic range of measurement systems.

- The instrumentation should provide information to an equipment protection system. This system must respond quickly to limit damage from beam losses.
- Large beam intensity at rapid pulse rate causes high radiation in the tunnel. Electronics located there should be kept to a minimum to avoid failures and minimize personnel exposure.

- The expected vacuum in the ring is $1 \times 10^{-7}$ Torr. Devices exposed to vacuum may require baking at up to $150^{\circ} \mathrm{C}$.

\section{3. $400 \mathrm{MeV}$ INJECTION LINE}

One Beam Position Monitor (BPM) and Beam Loss Monitor (BLM) will be installed at each of the 89 quads in Line. Beam Profile Monitors (BPrM) will be installed at every other quad and one Beam Current Transformer (BCT) will be placed at each end of the Line.

\subsection{BPM `s}

A shorted stripline design will be used to measure the 201.25 MHz frequency content of the beam. The signals will be transmitted through phase matched 1/2" Heliax cables to AM/PM conversion receivers. The bandwidth of the position output must be sufficient to track the 7.5 $\mathrm{MHz}$ chop rate. Position error from the BPM, cables, and electronics should be less than $\pm 0.5 \mathrm{~mm}$.

\section{$3.2 \mathrm{BLM}$ 's}

Sealed, argon-filled, glass ionization tubes [1] will be used as BLM `s. Typically, these monitors have 1 or 2 $\mu \mathrm{sec}$ response for electrons, $70 \mathrm{nC} / \mathrm{Rad}$ sensitivity, and are linear up to thousands of Rad/s. Such features are attractive for monitoring beam losses with one turn time resolution. Faster processing for a few monitors located near the injection area would be useful. The fast monitors should have programmable thresholds to provide alarm signals for the Equipment Protection System (EPS).

\section{$3.3 \mathrm{BCT}$ 's}

Two BCT's will monitor beam line intensity, transfer line efficiency, and injection efficiency for each injected turn. The $3.4 \times 10^{13}$ total intensity must be measured to $0.2 \%$ accuracy to verify that beam loss does not exceed the limit. This requires a time constant of more than 50 ms. A $20 \mathrm{MHz}$ high frequency response would resolve the time structure of the $7.5 \mathrm{MHz}$ chopped beam. The sensitivity will be $1 \mathrm{~V} / \mathrm{A}$ into $50 \Omega$. These bandwidth and accuracy requirements demand special attention.

\subsection{BPrM's}

To measure beam transverse distribution as well as horizontal and vertical emittances of the injected beam, use will be made of beam profile monitors based on movable second emission grids, slow wire scanners, and for continuos monitoring beam sizes Ionization Profile 
Monitors (IPM), one per plane, with MCP amplifier [2,3]. Heating of grid electrodes at full beam intensity and unwanted stripping of the ions are main topics of care.

\section{SYNCHROTRON}

Instrumentation for fast cycling high intensity machines should be capable of making measurements on turn-by-turn and, if possible, bunch-by-bunch basis. Assume one BPM and BLM for each of the approximately 100 quads. Tunes of 11.4 will provide just over 4 BPM's per betatron wavelength in each plane. Three IPM`s will be used to measure profiles on a turnby-turn basis. One IPM per plane at low dispersion locations and one horizontal IPM at a high dispersion location will allow measurement and correction for momentum spread. Two BCT's with different frequency ranges will be used in the ring. A fast $\mathrm{BCT}$ will measure injection efficiency with turn-by-turn resolution. It should resolve chopped beam and provide bunch-bybunch extraction efficiency in Stage 2 operation. A slow BCT will measure intensity and acceleration losses with higher resolution. Tune measurement systems, a wideband Resistive Wall Current Monitor (RWCM), and large bandwidth horizontal and vertical striplines will also be installed. Diagnostic equipment such as a vector signal analyzer, network analyzer, and fast digital oscilloscope should be permanently installed and interfaced to the controls system. Installing certain instrumentation such as the RWCM and large bandwidth striplines near the low level $\mathrm{rf}$ system would provide a central location and facilitate sharing resources.

\subsection{BPM's}

Elliptically shaped, diagonally split, electrostatic pickups (PU) will provide both good linearity and low beam impedance. The BPM system should provide turn-by-turn position information for all 100 BPM's on each of the 15,000 turns. Bunch-by-bunch measurements for Stage 2 operation may prove useful. The closed orbit would be determined by averaging several turns. Electronics based on the log-ratio technique [4] is preferred for its ability to accommodate various $\mathrm{rf}$ running modes and beam structures. Anticipated accuracy of the position measurement is better than $1 \mathrm{~mm}$ on a single turn.

A Beam Line Tuner (BLT) will measure turn-by-turn position at one horizontal and one vertical location and calculate betatron amplitudes and phases at injection to correct errors at injection. The BLT may require standalone position systems.

\subsection{BLM's}

The basic functions of the BLM system should be to minimize uncontrolled losses by providing data for tuning and disabling injection after high losses have occurred. About 110 monitors will be used: one downstream of each quadrupole and a few more at the injection, extraction, and collimation areas. Time response of $100 \mu$ s will be sufficient. BLM's in the injection and extraction areas may benefit from faster detectors and should be read with one turn time resolution. The BLM hardware would be similar to that used for the $400 \mathrm{MeV}$ Line. Programmable comparators will produce inputs to the EPS. They should not rely on software, as their reliability is critical.

\section{$4.3 B C T$ 's}

Both fast and slow BCT's will be based on toroidal cores of high permeability amorphous alloy. A core with dimensions $245 \times 295 \times 22 \mathrm{~mm}^{3}$ would fit around the 9" beam pipe. The fast BCT should provide: $0.1 \mathrm{~ms}$ time constant $(1.5 \mathrm{kHz})$, sensitivity of $1 \mathrm{~V} / \mathrm{A}$ into $50 \Omega$, and $15 \mathrm{~ns}$ rise time $(10 \mathrm{MHz})$. To achieve high noise immunity, careful attention to grounding and shielding will be necessary. Processing electronics for the fast BCT has challenging requirements: large dynamic range, necessity of base line restoration, variation of beam structure, and fast data flux. Variable gain, base line restoration, and an automatic calibration system will be required to measure turn-by-turn intensities to $0.2 \%$. Delivered data includes turn-by-turn: intensity, injection efficiency, and extraction efficiency. The slow DC Current Transformer should have $1 \mu \mathrm{A}$ resolution, $15 \mu \mathrm{A}$ noise, $50 \mu \mathrm{A}$ long term drift, and $4 \mathrm{kHz}$ bandwidth. An 18-bit A/D converter would provide $8 \mu \mathrm{A}$ resolution (1.6 $\times 10^{8}$ particles at injection) with a 2 Amp range $(3.0 \times$ $10^{13}$ at extraction). To verify efficiency before full power operation a minimum of $1.6 \times 10^{12}$ is required to obtain $10^{-4}$ resolution. The signal should be digitized at $1 \mathrm{kHz}$ or faster. Data processing must account for the changing beam velocity as it is accelerated.

\subsection{IPM}

The ring IPM's will be similar to those already in use at FNAL $[2,3]$. Care will be required when locating and using the monitors. Tunnel locations should be chosen to minimize damage of MCP's from radiation and X-rays. High output currents would quickly deplete the MCP's. Profile measurements should be done sparingly and plate voltage must be switched off immediately after the measurement. Because of faster drift times, turn-by-turn measurements may benefit from electron collection rather than the heavier ions. In this case, a magnetic field of 0.1 Tesla parallel to the electric field is necessary to reduce profile spreading caused by beam space charge. The collector arrays will consist of 64 strips with $1.0 \mathrm{~mm}$ spacing for vertical and $1.5 \mathrm{~mm}$ for the horizontal IPM`s. Electronic design and data handling would be similar to IPM's developed for the FNAL Booster [3].

\subsection{RWCM}

To observe the evolution of bunch shape and phase through the acceleration cycle, a wide-band RWCM will be installed. The low frequency limit is about $3 \mathrm{kHz}$, the microwave cut off frequency for the $5^{\prime \prime} \times 9^{\prime \prime}$ beam pipe is estimated to be $650 \mathrm{MHz}$. Low loss 7/8" Heliax cable 
short as possible should be used to minimize dispersion. A commercially available digitizer interfaced with the controls system will provide general measurements. The RWCM signal will be used by the Sampled Bunch Display and by the Fast Bunch Integrator to automatically track bunch intensities and shapes [5].

The RWM should be located near the Low Level rf System to allow its use for the phase lock loop. This will insure the availability of an accurate low noise clock for measuring bunch-by-bunch intensities. The RWM would be useful for monitoring $\mathrm{rf}$ capture at injection as well as bunch compression at the extraction energy.

\subsection{Fast Striplines}

Two, $1 \mathrm{~m}$ long, shorted, $50 \Omega$ stripline BPM's will be installed for general-purpose diagnostics. The expected bandwidth is about $660 \mathrm{MHz}$.

Low loss cables will transport the signal to hybrids located in the equipment gallery to produce $\Sigma / \Delta$ signals.

\subsection{Tune Measurement}

Tune measurements for both planes will be based on forced excitation of coherent transverse oscillations by a small angle kicker. An electrostatic PU one-quarter betatron wavelength downstream of the kicker will monitor these oscillations. The tune system should work with a few tenths of a millimeter kick to avoid emittance growth. The $\Delta$ signal of the PU should have $250 \mathrm{kHz}$ bandwidth centered between rotation harmonics. Considering the change in rotation frequency in the ring, using one of the lower rotation harmonics may be desirable. An FFT of 256 samples taken once per turn will provide 0.004 tune resolution. Both kickers will be 1 $\mathrm{m}$ long $50 \Omega$ striplines.

\subsection{Transverse Damper}

Transverse bunch-by-bunch active damping systems will be used to damp injection oscillations and coherent instabilities. These systems would be built relying on existing Dampers at FNAL [6,7]. The dampers will consist of four basic parts: the stripline PU`s, processing electronics, power amplifiers, and stripline kickers. Two important features are a $90^{\circ}$ betatron phase advance between the PU and kicker locations and a time delay through the processing electronics which matches the beam flight time. The betatron phase advance will depend on the tune of the machine. To accommodate the 3.3 to $2.4 \mu$ s change in rotation period, the processing electronics must incorporate a compensating variable delay. To obtain the required damping gain without overdriving the amplifiers, notch filters designed to remove energy at the rotation harmonics are required. Digital filters clocked in synch with the accelerating rf are used to form the notch filters. One feature of this approach is that the notches and the delay automatically track the revolution period. An additional phase correction may be required to account for the frequency dependent phase shift from pickup and kicker cables.

\section{16 GeV EXTRACTION LINE}

As in the $400 \mathrm{MeV}$ line, one BPM and BLM will be installed at each of the 65 quads in the $12 / 16 \mathrm{GeV}$ Line. BPrM`s will be installed at every other quadrupole and one BCT will be placed at each end of the Line. BLM's and BPrM's will be similar to the $400 \mathrm{MeV}$ Line. BCT's and BPM's would be similar to those used in the PD ring. Upstream of each extraction magnet and at the exit window, four BPM's will measure radial position during extraction. Two bi-plane movable BPM`s will be mounted upstream of the first septum and at the exit window. Three BLM's will be placed downstream of each extraction magnet and one close to the exit window. The four extraction BPM's and the upstream BCT should be able to resolve changes during the $2.4 \mu$ s extraction turn, other devices would be sampled once per extraction.

\section{EQUIPMENT PROTECTION SISTEM}

The purpose of the EPS is to protect equipment and provide a safe means of increasing beam intensity while starting up the accelerator. The EPS will monitor selected devices and signals and inhibit subsequent injections or abort the beam when they are out of tolerance. Excessive beam loss, poor efficiency, bad vacuum, insufficient rf voltage, and key equipment status will likely be monitored. Indications of excessive coherent transverse oscillations from the damper or failures in the timing system may also be used. The EPS should be cast in hardware and consist of a permit loop with general purpose input chassis at key locations in the equipment galleries. The EPS system should respond in less than 100 $\mu$ s. Hardware limits should be remotely controllable in a fashion easy to monitor and maintain.

\section{REFERENCES}

[1] R.Shafer, et al., "The Tevatron Beam Position and Beam Loss Monitor System", Proc. $12^{\text {th }}$ Int. Conf. On HEA, p.609 (1983).

[2] Zagel, at al., "Fermilab Main Ring Ion Profile Monitor System", PAC" 97, p.2166 (1997).

[3] J.Zagel, D.Chen, and J.Crisp, "Fermilab Booster Ion Profile Monitor System Using LabVIEW," AIP Conf. Proc.333, p.384.

[4] G.Aiello, M.Mills, R.Gonzalez, "Test Results of the SSC Log-Ratio Beam Position Monitor Electronics", PAC'93, p.2322 (1993).

[5] J.Utterback, G.Vogel, "System for Measuring Bunch Intensities in Fermilab's Main Ring”, Proc. ICALEPCS95, p.628 (1995)

[6] J.Crisp, et al., "A programmable High Power Beam Damper for the Tevatron", PAC85, p.2147 (1985).

[7] J.M.Steimel Jr. and D.McGinnis, "Damping in the Fermilab Booster", PAC93, p.2100 (1993). 